\title{
Nanotechnology in dentistry
}

\author{
Melanie Sadono Djamil \\ Biochemistry Department \\ Faculty of Dentistry Trisakti University \\ Jakarta - Indonesia
}

\begin{abstract}
Technology has continuously improved along with the complexity of devices. Nowadays, it is widely accepted that micro-technology, which is defined as a further reduction in the size of interconnections and components, is achieved by a conventional "top-down" method. We have now moved to a new concept and approach for fabrication from small to bigger building-block elements, which is called nanotechnology. Nanotechnology is the fabrication technology of tiny parts that is achieved by a "bottom-up" method. Nanotechnology has been developed in many areas of life sciences, such as in dentistry. This presentation provides some examples that illustrate the progress in technological growth, especially in the nanoscale. In the developments of nanotechnology, we are also concerned in many ways about its ethics and the laws of physics. The expansion in nanotechnology shows that much multidisciplinary research is being done in the nanoscale area. In dentistry, one of the examples is research in dental materials such as nanoleakage types in the use of various adhesives with resin composition. Nanodiagnostics are nanotechnology in applied molecular diagnostics. All these fields have applications in diagnostics and in point-of-care hand-held devices.
\end{abstract}

Key words: nanotechnology, nanoscale, nanodiagnostics, nanoleakage

Correspondence: Melanie Sadono Djamil, c/o: Bagian Biokimia, Fakultas Kedokteran Gigi Universitas Trisakti. Jln. Kyai Tapa, Grogol Jakarta, Indonesia. E-mail: melaniehendriaty@trisakti.ac.id

\section{INTRODUCTION}

In the middle of the $20^{\text {th }}$ Century, a new technology was developed in various fields of science called microtechnology. In the ensuing progress at the beginning of the $21^{\text {st }}$ Century, a technological revolution occurred that began with new developments in the technology of computers and the Internet, namely nanotechnology. Nanotechnology has since colored research and technological developments in engineering, basic sciences, medicine, dentistry, and even social sciences.

The methods of fabrication of materials and devices are today known through the molecular level: casting, grinding, milling up to the lithographic movement of a group of atoms. As if we wanted to make a Lego construction with our hands in boxing gloves: you cannot really snap the pieces together the way you like. ${ }^{1}$

In the future, nanotechnology will allow us to be free of those boxing gloves so that we can readily build from the base the construction that we want, which is strong and easy in terms of the laws of physics that indeed enable this to be possible. Similarly with the developments in computer science in coming decades, it may be possible to make a new generation of computers that have a clearer resolution, are stronger, lighter and more precise. ${ }^{1}$

The trends in nano-scale research are getting brighter and expanding to such an extent that discussions in all branches of knowledge are heading towards nano-science and nanotechnology.
What follows in this paper is a discussion of the formation of organic and inorganic materials, as well as hybrid materials, on a nano-scale through methods of chemistry, physics and biology, as well as involving nanostructures and nano-devices. ${ }^{2}$

The position of nanotechnology and its affect on all branches of science and life are very significant, particularly where the aim of such science is to change a situation that is not good or beneficial into something better. An example of such assistance from nanotechnology in the community is water filtering systems using nanotechnology that produce better sources of water and better sanitation. ${ }^{2}$

In the basic sciences, particularly molecular biology, there have been 50 years of developments in DNA structure, which was introduced by Watson and Crick in 1954. It was 20 years later when their work got acknowledged with the Nobel Prize. It began with a central dogmatic theory that was followed later by scientific advances in genetics, which originated in biological phenomena such as theories of recombination and growth. ${ }^{2}$

Initially, there was an understanding of the formation of a product made from atomic elements, and how those atoms were structured. If we recombine the atoms of coal, we can create diamonds; if we recombine the atoms of sand (and adding other trace elements), we get computer chips. If we recombine the atoms of feces, water and air, we get potatoes. ${ }^{1}$ 


\section{Nanotechnology of DNA Structure}

DNA is more than merely the secret of life because it is a versatile component in the formation of structures or devices. In 2003, we had reached 50 years since the double-chain structure of DNA was discovered. Their discovery is the basis of genetical chemistry and biology. Now, many researchers have proven that genes can affect the development and growth of organisms. In fact, DNA has functions other than in biochemistry, such as in modern biotechnology where the long DNA chains can be used as computer materials. This is called a non-biological use of DNA. ${ }^{3}$
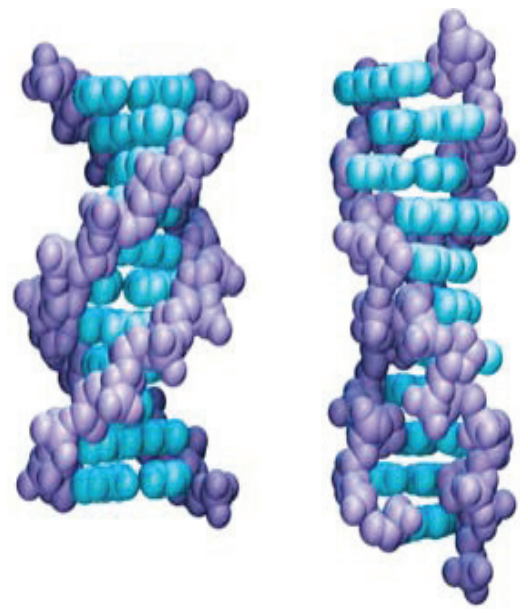

Figure 1. An example of a nano-scale DNA structure, comprising of a pair of phosphate ions and sugar molecules that are paired with complementary base A \& $\mathrm{T}$ and $\mathrm{C} \& \mathrm{G}$, by a weak bond.

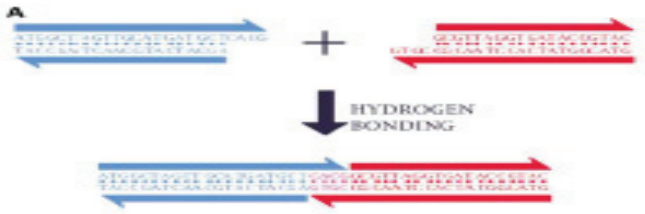

B

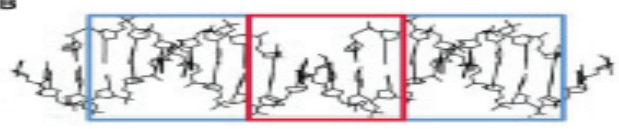

c
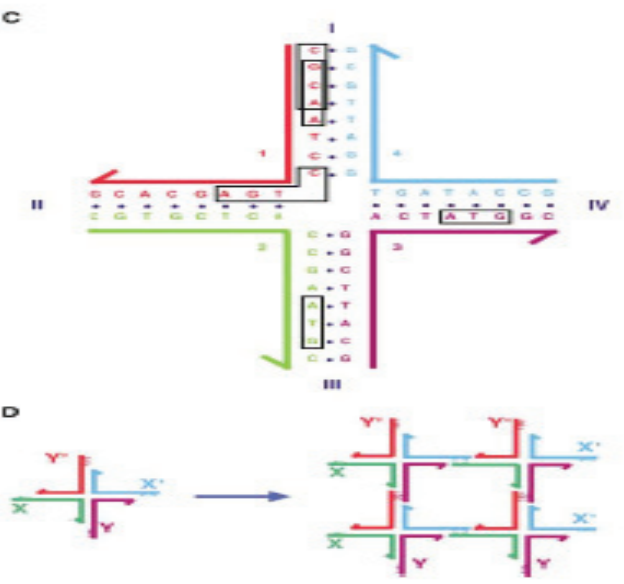

Figure 2. Nanotechnology of basic DNA structure.
DNA is an ideal molecule with a structure of a nanometer scale; the chains can form themselves into a complex form of double chains each with its own complement. The main aim of DNA-based nanotechnology is a continuation of two-dimensional success to be threedimensional. Basically, nanotechnology of DNA structure is a concept of combining the stable DNA branches with the ends adhering cohesively (Figure 2$)^{2}$

\section{Nanomachines}

The centre or core of nanotechnology is a molecularscale machine. DNA has proven to play a decisive role in the formation of such a machine. Several machines/devices have been made originating from DNA. The mechanism of their formation is based on a transition of the structure of DNA molecules, such as a change of the conformation from a double-helix form into another shape. ${ }^{4}$

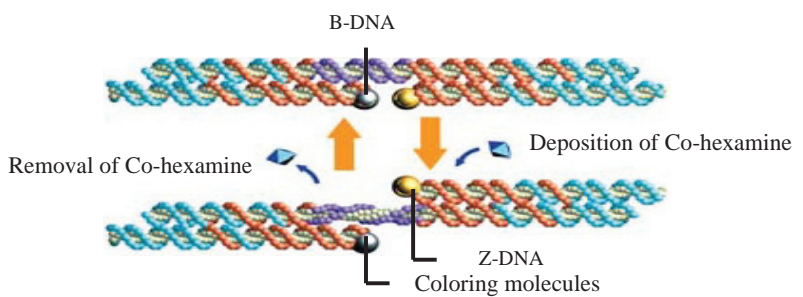

Figure 3. Nanomechanical B-Z Device. Shows controlled movement from the two double helixes (blue and orange) that are connected by a hand comprising 20 alkali pairs (purple).

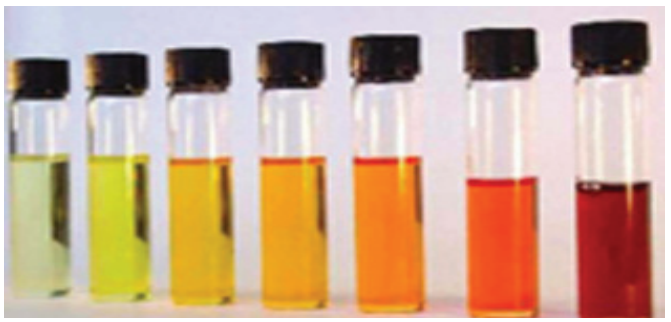

Figure 4. Semi-conductor solutions of same material (CdSe), where different colors are given off due to the size of the particles.

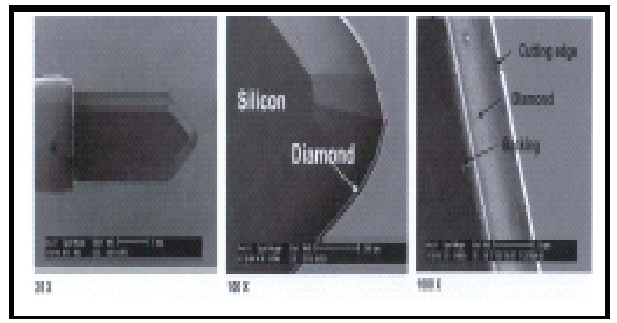

Figure 5. Surgical knife made from microstructured-silicon with a diamond-layered tip.

Conventional DNA is a helix that twists to the right (right-handed DNA structure), which we can imagine as if we were climbing a spiral staircase with our left hand 
holding the inside handrail and our right hand holding the outside handrail. The conventional DNA is called B-DNA, is soluble in water and has high energy. ${ }^{2}$

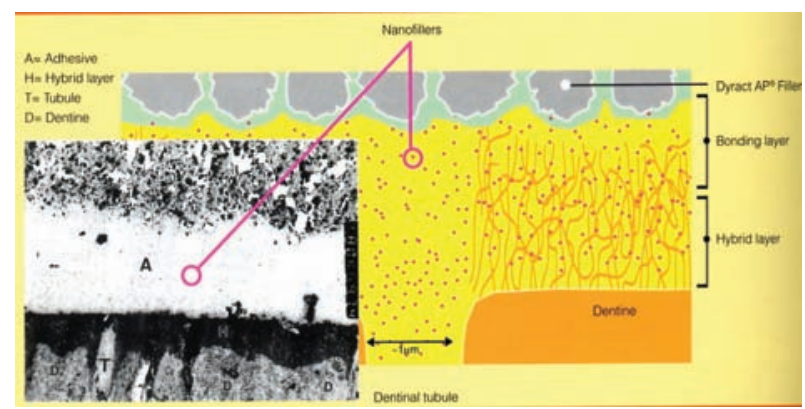

Figure 6. Teeth filled with the latest generation fillings (nanofillers and nano-hybrids).

In addition to that, since 1979, Alexander Rich and colleagues at $\mathrm{MIT}^{1}$ have known of Z-DNA that spirals to the left (left-handed DNA structure). One of the DNA structures of the spine contains negative phosphate groups that approach each other in the Z-DNA structure area. They contain a lot of cytosine and guanine alkalis. ${ }^{2}$

\section{Nanomedicine}

In years to come, the development of nanotechnology applications is going to be quite intensive, including in the fields of health. Nanotechnology has not only attracted scientists but also governments, academics and industry, even though it is difficult to give an exact definition of 'nanotechnology', and where it differs from microtechnology. It is most likely that the defining point is the difference in their formation, namely micro-structures are formed by a "top down" process while nano-structures are formed through a "bottom up" process. ${ }^{5}$

Micro-technology can be defined as the formation of structures through a "bottom up" process of the bulk deposition and removal of a material, as occurs in a microelectronic industry. This is seen as a "top down" approach resulting in a final structure, which begins with materials of a large physical size. ${ }^{6}$

Conversely, nanotechnology is the formation of a structure through a "bottom up" process, starting at the molecular level and using atoms or molecularscale materials, which results in the desired structure. Nanotechnology is not merely a 'laboratory curiosity technology'. Such a view has circulated in the markets, such as with sunscreens that have nano-particles added, window panes made with nano-engineering so that dirt does not easily stick to them, and wall paint that changes its color when viewed from different angles. Since 2003 in America, research into nanotechnology itself has consumed more than USD700 million. Health sciences and biomedicine are the prime targets. ${ }^{6}$

Evaluation of nano-particle biology has been around long, and so will be soon be familiar and cleaned of its macrophage Pharmaco-kinetic medicine is layered with nano-particles and given intravenously so that the length of time of the medicine undergoes a change. The pharmacokinetics of medications and the biological characteristics of the particles are therefore estimated to remain long in the blood circulation, whether they need to be administered once or repeatedly, needs to be designed precisely so that the optimal effect of the medicine, for both therapy and diagnostics, is achievable. ${ }^{7}$

\section{Phosphorus and biolabels}

Nano-particles have physical characteristics that are generally very different from the bulk material. An example is quantum dots whose electronics is set by the theories of quantum physics: if they are hit by light of a specific wave length, they will emit light at a different wave length, which is related to the size of the particles. The picture below shows solutions containing the same semi-conductor material $(\mathrm{CdSe})$, but produce different colors; this is caused by the size of the particles. ${ }^{8}$

Oxonica (Oxford, UK) has developed a phosphorous material that emits color of a narrow wave length. This can be used as a replacement material/solution for use in diagnosis (where luminescent organic dyes are normally used) with high stability, non- toxicity, all colors readable at a single wave length, colors produced being stable at specific times, the screening process can be automated - because the output spectrum of nano-particles have sharp biolabels and high quality results. ${ }^{9}$

\section{Healing of wounds}

Nucryst (Wakefield, USA) has made a wound-healing material (dressing) that is generally used in specialist burn-treatment hospitals in America. The dressing contains nanocrystalline silver that stops 150 types of fungus and bacteria, including several bacteria that are resistant to antibiotics. Meanwhile, another company has placed nanoparticles into a plastic material to make it biocidal. This can be useful for devices that are placed inside the body. ${ }^{10}$

\section{Batteries for portable devices}

Batteries can be modified by nanotechnology. Ntera (Dublin, Ireland) has developed a nano-scale low-cost refillable battery material of ion lithium. The metal oxide in the new nano-structure will replace the traditional type of electrode material in ion lithium batteries, which can be refilled several times. ${ }^{4}$

\section{Surgical devices}

The characteristics of materials change drastically according to the thickness of some molecules. GFD (Ulm, Germany) has produced a surgical knife from microstructured-silicon with a diamond-layered tip (see picture below). Diamond is a material that is chemically rigid, and silicon is non-magnetic and biocompatible. The knife therefore makes sharper incisions and with a lower penetration pressure. ${ }^{11}$ 


\section{Implants}

Nanotechnology has enabled the creation of new surfaces, visual effects and overall changes that are beneficial. Sus Tech GmbH (Darmstadt, Germany) has worked on nano-apatite and other biocomposites for the surface of tooth and bone prostheses. Generally, the use of artificial spine implants leads to irritation for the user because the surface is not hard enough, so by making the surface tougher, the life of the implant is extended. ${ }^{6}$

\section{Nano-capsules}

Nanotechnology can assist in the precise delivery of medicine to the target organ. Layered with nano-scale polymer particles, materials become hydrophilic and immune to natural bodily mechanisms. During the past five years, hundreds of fabricated protein medicines have reached the market, for example, insulin, growth factor supplements, cytocines, and monoclonal antibodies. Protein itself cannot be taken orally because the digestive system will break it down into amino acid and the protein molecules are too large to be given transdermally. Consequently, the administration of such medicines by inhalation is very necessary. ${ }^{10}$

Nano-crystals (King of Prussia, Pennsylvania, USA) allow the provision of medicines in various forms. Medicines in nano-crystal form are more readily absorbed. This new technique stabilizes the medicine particles with polymer on its surface so that it changes clinical patches and medication that has a bad consistency. ${ }^{4}$

FeRex (San Diego, California, USA) has been increasingly developed and marketed in the technological form of Magnetic Target Carriers (MTC) to specifically reach a target site and to enable the release of the pharmacological materials that can be absorbed by the MTCs. $^{4}$

\section{DISCUSSION}

In dentistry nanotechnology have been used, one of the examples in dental materials. Resin composites that are combined with particles of nano-fill resin composites (Supreme) and nanohybrid (Grandio) plus an ormocerbased tooth-colored restorative material (Admira) after the finishing and polishing procedure will improve the rough surface. These days, patients can choose the treatment that is going to be done on their teeth, especially from an esthetic point of view, as well as biocompatibility, durability, length of use, and safety. Posterior esthetic fillings are generally not so good from a biological and physio-chemical point of view compared to gold and amalgam. First generation composite resins were limited in their resistance to the use abrasions, and their color varied; they shrank after polymerization, had low strength and elasticity, and were easily fractured. New formulas-types of nano-fillers-reduce the shrinkage after polymerization, increase the strength and elasticity, reduce the fractures, and can withstand abrasion and congestive discoloration.
In addition, various combinations of light, heat, pressure, vacuums and nitrogen are used to increase the degree of strength after the post-curing period and repair the physical characteristics and mechanics of the resin. Other additional results of the latest generation fillings are their optical characteristics that are more translucent, fluorescent and opalescent so that they resemble the structure of real teeth. $^{12}$

Esthetic dentistry has been expanding through innovations in bonding systems, restorative materials, function-based treatments, and the design of conservation preparations. The latest generations of direct resin composites for posterior teeth are very beneficial for certain cavities, particularly those that are not too wide, or just small cavities, keeping in mind those shrinkages still occur. $^{13}$

Indirect resin composite systems are more recommendable for large cavities. These indirect systems can satisfy the mechanical and biological requirements, while also achieving esthetic results, and shrinkage is less and more easily manageable. The materials used, which are called ceramic optimized polymers, can maintain a high density of inorganic ceramic micro-fillers compared to previous generations. These second-generation indirect systems are known as resin or a porcelain composite whose limitations cannot be overlooked but the value of their use is high. ${ }^{13}$

The materials can be classified as 'micro-hybrids' that include combinations of inorganic particles (as fillers) and organic polymers (matrix) with a ratio of $2: 1$. The fillers are a main determining material of the clinical and physiochemical characteristics of a resin composite. The particles of the sub-micron fillers can determine the characteristics of the surface, such as the polishing capacity and wear resistance. The use is affected by the size of the fillers, and the shape, burden and bonding of the matrix. ${ }^{12}$

As additions, there are diverse combinations of light, heat, pressure and vacuums, as in the use of nitrogen, for speeding up the process after curing in order to improve the physical characteristics of the second generation indirect resin systems. The curing process removes the monomer remnants, and enables the optimal polymerization to be achieved. Oxygen is removed through a vacuum process, and pressure or nitrogen can remove trapped air, so that the opacity of the restorative material can be preserved. Precise and good manipulation can result in maximum strength and homogeneity, esthetic qualities, color stability, and extend the strength of the material in use. ${ }^{12}$

Based on the results of research done by Suzuki ${ }^{14}$ by comparing several denture materials using nano-filled filling material (Veracia) with micro-filled dentures (SROrthosit, Endura, Duardent, Surpass), cross-linked acrylic (SR-Postaris, Genios-P, Creapearl, Vitapan Physiodens, Premium 8, Integral), it was proven that dentures with basic materials of nano-composites were stronger and more wear resistant compared to using acrylic, but almost the same as micro-filled dentures. ${ }^{14}$ 
The in-vitro studied use of the developments of nano-filler and nano-composite filling materials to other composites such as hybrids, micro-hybrids and microfillers. The researches concluded that nano-fillers and nano-composites were stronger and esthetically better with the translucent characteristics of the teeth, and are usable for both anterior and posterior teeth.

With the increasing developments of bionanomaterial science, we are forced to go into it more deeply both theoretically and through research so that we can understand the structures and functions of bionanomaterials. We also need to look into the use of DNA, RNA and peptides as the basis of the developments in biology and nanomaterials. Particularly deserving of our attention are biochemistry, biophysics, thermodynamics, and the electronic content of DNA, RNA and peptides because they represent important matters in interdisciplinary life sciences and material sciences.

There are many benefits from studying and using nanobiochip materials, interface materials and nanobiosensors, as well as systems of nano-drug-delivery, in industry, resistance, and applications in health sciences. We therefore need to always review our current uses so that the developments of bionanomaterial technology can be beneficial for the whole world.

\section{REFERENCES}

1. Doty RC, Fernig DG, Levy R. Nanoscale science: a big step towards the Holy Grail of single molecule biochemistry and molecular biology. Cell Mol Life Sci 2004 Augustus; 61(15):1843-9.
2. Seeman NC. Biochemistry and structural DNA nanotechnology: an evolving symbiotic relationship. J Biochemistry 2003 June 24; 42(24):7259-69.

3. Minguez N, Ellacuria J, Soler JI, Triana R, Ibaseta G. Advances in the history of composite resins. J Hist Dent 2003 November; 51(3):103-5.

4. Allen KL, Schenkel AB, Estafan D. 2004. An overview of the CEREC 3D CAD/CAM system. Gen Dent. May-Jun; 52(3):234-5.

5. Carella M, Volinia S, Gasparini P. Nanotechnologies and microchips in genetic diseases. J Nephrol 2003 July-Augustus; 16(4):597-602.

6. Silva GA. Introduction to nanotechnology and its applications to medicine. Surg Neurol 2004 March; 61(3):216-20.

7. Williams D. Nanocrystalline metals: another opportunity for medical devices? Med Device Technol 2003 November; 14(9):12, 16-7.

8. Vo-Dinh T. Nanobiosensors: probing the sanctuary of individual living cells. J Cell Biochem 2002; 39(Suppl):154-61.

9. Jain KK. Nanodiagnostics: application of nanotechnology in molecular diagnostics. Expert Rev Mol Diagn 2003 March; 3(2):153-61.

10. Huikko K, Kostiainen R, Kotiaho T. Introduction to micro-analytical systems: bioanalytical and pharmaceutical applications. Eur J Pharm Sci 2003 October; 20(2):149-71.

11. Wilkinson JM. Micro- and nanotechnology fabrication processes for metals. Med Device Technol 2004 June; 15(5):21-3.

12. Burgess JO, Gallo JR, Ripps AH, Walker RS, Ireland EJ. Clinical evaluation of four Class 5 restorative materials: 3-year recall. Am J Dent 2004 June; 17(3):147-50.

13. Tay FR, Pashley DH, Yiu C, Cheong C, Hashimoto M, Itou K, Yoshiyama M, King NM. Nanoleakage types and potential implications: evidence from unfilled and filled adhesives with the same resin composition. Am J Dent 2004 June; 17(3):182-90.

14. Terry DA, Leinfelder KF. An integration of composite resin with natural tooth structure: the Class IV restoration. Pract Proced Aesthet Dent 2004 April; 16(3):235-42. quiz 244.

15. Mitra SB, Wu D, Holmes BN. An application of nanotechnology in advanced dental materials. J Am Dent Assoc 2003 October; 134(10):1382-90 